\title{
Case Report: Efficacy of Ninjin'yoeito Treatment for Idiopathic Pulmonary Fibrosis
}

\author{
Hisako Kushima ${ }^{1 *}$, Hiroshi Ishii ${ }^{2}$ and Masaki Fujita ${ }^{1}$ \\ ${ }^{1}$ Department of Respiratory Medicine, Fukuoka University Hospital, Fukuoka, Japan, ${ }^{2}$ Department of Respiratory Medicine, \\ Fukuoka University Chikushi Hospital, Chikushino, Japan
}

Ninjin'yoeito, a Kampo prescription, was administered to two patients with idiopathic pulmonary fibrosis (IPF) over a period of 12 weeks to improve loss of appetite and lassitude. In Case 1, improvements were observed in appetite, lassitude, and breathlessness, as well as increases or increasing tendencies in body weight, blood albumin level, and hemoglobin $(\mathrm{Hb})$ level. Case 2 showed no changes in appetite but improvements in lassitude and no deterioration of breathlessness. His body weight and his blood albumin and $\mathrm{Hb}$ levels increased or showed increasing trends. In both cases, a trend for improvement of respiratory function was observed. In summary, ninjin'yoeito trended to improve the subjective symptoms and nutritional status of a patient with pulmonary fibrosis.

Keywords: idiopathic pulmonary fibrosis, loss of appetite, malaise, ninjin'yoeito, Kampo medicine

\section{OPEN ACCESS}

Edited by:

Akio Inui,

Kagoshima University, Japan

Reviewed by:

Tomoo Kishaba,

Okinawa Chubu Hospital, Japan Keiko Ochiai Ogawa,

Kanazawa University, Japan

*Correspondence:

Hisako Kushima

hkushi@fukuoka-u.ac.jp

Specialty section

This article was submitted to Clinical Nutrition

a section of the journal

Frontiers in Nutrition

Received: 01 April 2020

Accepted: 29 March 2021

Published: 29 April 2021

Citation:

Kushima H, Ishii H and Fujita M (2021)

Case Report: Efficacy of Ninjin'yoeito

Treatment for ldiopathic Pulmonary Fibrosis. Front. Nutr. 8:548076. doi: 10.3389/fnut.2021.548076

\section{INTRODUCTION}

Idiopathic pulmonary fibrosis (IPF) is a chronic and progressive disease in which advanced fibrogenesis results in irreversible honeycomb lungs. Antifibrotic agents can be used to treat IPF but the efficacy is limited. The mean lifespan after diagnosis of IPF is 3-5 years; and the mean lifespan after exacerbation of IPF is 2 months or less. IPF is associated with a very high mortality rate (1). Accompanying the progression of restrictive ventilatory defect, the IPF patient may complain of subjective symptoms, such as, exertional dyspnea, dry cough, weight loss, malaise, and/or fatigability. Since these symptoms significantly reduce patients' quality of life, we decided to administer ninjin'yoeito as an available medicine for malaise under universal health coverage in Japan. This report describes the administration of ninjin'yoeito to 2 IPF patients who had experienced loss of appetite and malaise, and a clear therapeutic response was observed.

\section{CASE REPORT}

Case 1 was a 59-year-old male with a history of rhabodomyolysis. The patient was referred to our hospital because of gradually progressive dyspnea on exertion. His height was $155.5 \mathrm{~cm}$, the body weight was $51.8 \mathrm{~kg}$ and the percentage of oxygen saturation ( $\mathrm{SpO} 2)$ was $95 \%$. His respiratory test results are shown in Table 1. Chest computed tomography (CT) demonstrated subpleural fibrotic opacities with traction bronchiectasis in the upper and lower lung fields. He was clinically diagnosed as IPF with pleuroparenchymal fibroelastosis-like lesions based on the guidelines (2) (Figure 1). He was treated with nintedanib ethanesulfonate and $7.5 \mathrm{~g}$ (divided into two doses) of ninjin'yoeito. The appetite score [Simplified Nutritional Appetite Questionnaire, SNAQ $(3,4)$ ] showed improvement, increasing from 15 before administration to 16 after both 4 and 12 weeks of administration (SNAQ consists of four items related to appetite. All questions are scored from 1 to 5 points, with a total score ranging from 4 to 20 points. The lower the score, the poorer the appetite). Similarly, the 
TABLE 1 | The results of pulmonary function tests and biomarkers before and after ninjin'yoeito treatment in Case 1.

\begin{tabular}{lcc}
\hline & $\begin{array}{c}\text { Before } \\
\text { administration }\end{array}$ & $\begin{array}{c}\text { After 12 weeks of } \\
\text { administration }\end{array}$ \\
\hline Vital capacity (VC) (L) & 2.18 & 2.45 \\
\%Vital capacity (\%VC) & 63.2 & 72.5 \\
Forced vital capacity (FVC) (L) & 2.14 & 2.42 \\
Forced expiratory volume 1.0 & 1.86 & 2.02 \\
(FEV1) (L) & & 83.5 \\
Forced expiratory volume & 86.9 & 481 \\
$1.0 \%(F E V 1 \%)$ & & 181 \\
KL-6 (U/ml) & 499 & \\
LDH (IU/L) & 162 & \\
\hline
\end{tabular}

lassitude score [Chalder Fatigue Scale, CFS $(5,6)]$ improved from six before administration to four after 4 and 12 weeks of administration (CFS consists of 11 items related to physical and mental fatigue. All questions are scored from 0 to 3 points, with a total score from 0 to 33 points. The higher the score, the stronger the fatigue). The breathlessness score [Modified Medical Research Council dyspnea scale, mMRC (7)] also improved from two before administration to one after both 4 and 12 weeks of administration (mMRC scale uses a 5grade scale, with Grade 0 representing almost no breathlessness and Grade 4 representing severely impaired activities of daily living due to breathlessness. The higher the grade, the stronger the breathlessness). Body weight increased from $51.8 \mathrm{~kg}$ before administration to 54.5 and $55.2 \mathrm{~kg}$ after 4 and 12 weeks of administration, respectively. The blood albumin level increased from $3.6 \mathrm{~g} / \mathrm{dL}$ before administration to $4.5 \mathrm{~g} / \mathrm{dL}$ after both 4 and 12 weeks of administration, and the $\mathrm{Hb}$ level showed an increasing trend, changing from $14.9 \mathrm{~g} / \mathrm{dL}$ before administration to 15.6 and $15.4 \mathrm{~g} / \mathrm{dL}$ after 4 and 12 weeks of administration, respectively. The CONUT scores were zero before and 12 weeks after ninjin'yoeito administration. A trend for improvement was also observed with respect to respiratory function test results. Serum KL-6 decreased from 499 to $481 \mathrm{U} / \mathrm{ml}$. LDH increased from 162 to $181 \mathrm{IU} / \mathrm{L}$ (Table 1). There was no occurrence of transaminitis during the treatment. He had an increase in walking distance from 383 to $440 \mathrm{~m}$ in a 6-min walk test (6MWT) 12 weeks after administration. The value of the lowest SpO2 at $6 \mathrm{MWT}$ remained unchanged at $\sim 90 \%$ for 12 weeks. The CT finding did not show significant changes before and after treatment with ninjin'yoeito.

Case 2, a 59-year-old male was referred to our hospital for respiratory failure and was clinically diagnosed as IPF based on the guidelines [Raghu et al. (2)] (Figure 2).

His height was $167.5 \mathrm{~cm}$, the body weight was $50.2 \mathrm{~kg}$ and the $\mathrm{SpO} 2$ was $90 \%$. His respiratory test results are shown in Table 2. He was treated with nintedanib ethanesulfonate and $7.5 \mathrm{~g}$ (divided into 2 daily doses) of ninjin'yoeito. CFS decreased from 27 before administration to 16 and 24 after 4 and 12 weeks of administration, respectively, showing improvement. The mMRC scale was three before administration and 2 and

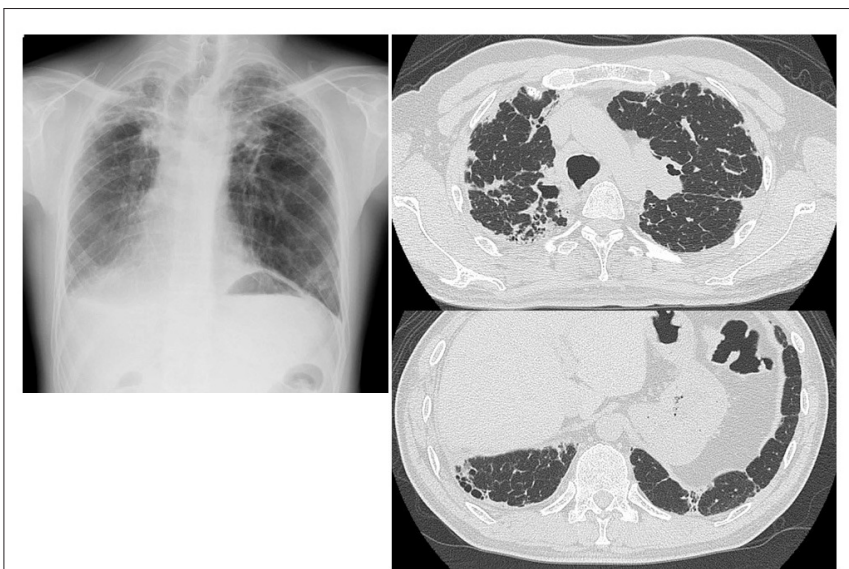

FIGURE 1 | Chest radiograph and CT scans of Case 1 showing elevation of bilateral hilar opacities and subpleural reticular opacities in the bilateral lung fields.

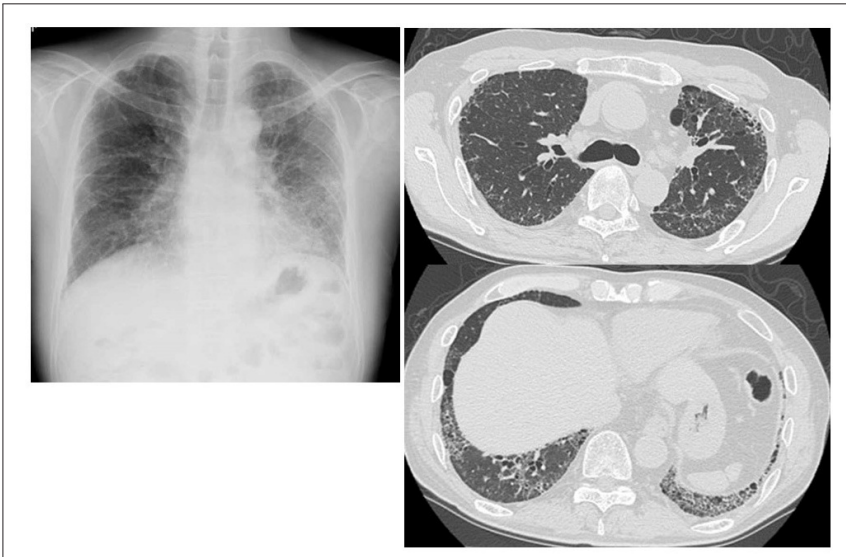

FIGURE 2 | Chest radiograph and CT scans of Case 2 showing reticular opacities, traction bronchiectasis, and some ground-glass attenuation in the bilateral lung fields.

3 after 4 and 12 weeks of administration, respectively, showing no deterioration. The SNAQ score was 14 before administration and after 4 and 12 weeks of administration, showing no change. Body weight increased from $50.2 \mathrm{~kg}$ before administration to 50.4 and $51.9 \mathrm{~kg}$ after 4 and 12 weeks of administration, respectively. The blood albumin level was $4.1 \mathrm{~g} / \mathrm{dL}$ before administration to 4.1 and $4.3 \mathrm{~g} / \mathrm{dL}$ after 4 and 12 weeks of administration, respectively, showing an increasing trend. The $\mathrm{Hb}$ level increased from $12.8 \mathrm{~g} / \mathrm{dL}$ before administration to 13.7 and $14.3 \mathrm{~g} / \mathrm{dL}$ after 4 and 12 weeks of administration, respectively. The CONUT score could not be measured because the serum cholesterol levels were not measured. The levels of albumin and lymphocyte counts were normal throughout the treatment period. A trend for improvement was also observed with respect to respiratory function. Serum KL-6 decreased slightly from 496 to $492 \mathrm{U} / \mathrm{ml}$. LDH decreased from 201 to 196 IU/L (Table 2). There was no occurrence of transaminitis during the treatment. A 6MWT after 
TABLE 2 | The results of pulmonary function tests and biomarkers before and after ninjin'yoeito treatment in Case 2.

\begin{tabular}{lcc}
\hline & $\begin{array}{c}\text { Before } \\
\text { administration }\end{array}$ & $\begin{array}{c}\text { After 12 weeks of } \\
\text { administration }\end{array}$ \\
\hline Vital capacity (VC) (L) & 1.65 & 1.90 \\
\%Vital capacity (\%VC) & 42.3 & 48.7 \\
Forced vital capacity (FVC) (L) & 1.62 & 1.83 \\
Forced expiratory volume 1.0 & 1.19 & 1.43 \\
(FEV1) (L) & & 78.1 \\
Forced expiratory volume 1.0\% & 73.5 & 492 \\
(FEV1\%) & & 196 \\
KL-6 (U/ml) & 496 & \\
LDH (IU/L) & 201 & \\
\hline
\end{tabular}

14 weeks of administration showed improvement in the lowest SpO2 (from 87 to 91\%) while the walking distance decreased from 360 to $300 \mathrm{~m}$. The CT finding did not show significant changes before and after treatment with ninjin'yoeito.

\section{DISCUSSION}

Ninjin'yoeito was administered to patients with IPF in this study. The treatment improved subjective symptoms such as, loss of appetite, malaise, and breathlessness, as well as scores related to nutritional status, including body weight and blood albumin and Hb levels. In a basic pharmacological study using a bleomycininduced pulmonary fibrosis model, ninjin'yoeito was reported to have suppressed decreases in food intake and body weight, atrophy of the gastrocnemius, and expression of inflammatory cytokines in the lungs (8). Regarding the mechanism by which it improves loss of appetite, activation of ghrelin-responsive, and unresponsive neuropeptide $\mathrm{Y}$ neurons in the arcuate nucleus of the hypothalamus (9) and actions mediated by dopamine $\mathrm{D}_{2}$ receptors (10) have been reported.

Ninjin'yoeito was administered to the two patients described herein to improve subjective symptoms, such as, loss of appetite and malaise. In addition to these improvements, trends for improvement were also observed with respect to respiratory function after 12 weeks of administration. Ninjin'yoeito is a Kampo prescription consisting of 12 crude drugs. Unlike

\section{REFERENCES}

1. The Japanese Respiratory Society. Guidelines for the Treatment of IPF 2017 (in Japanese). Tokyo: Nankodo (2017).

2. Raghu G, Remy-Jardin M, Myers JL, Richeldi L, Ryerson CJ, Lederer DJ, et al. Diagnosis of idiopathic pulmonary fibrosis. An official ATS/ERS/JRS/ALAT clinical practice guideline. Am J Respir Crit Care Med. (2018) 198:e4468. doi: 10.1164/rccm.201807-1255ST

3. Nakatsu N, Sawa R, Misu S, Ueda Y, Ono R. Reliability and validity of the Japanese version of the simplified nutritional appetite questionnaire in community-dwelling older adults. Geriatr Gerontol Int. (2015) 15:12649. doi: 10.1111 /ggi.12426 hochuekkito and juzentaihoto, which are Kampo prescriptions that are similarly used to improve lassitude and malaise, ninjin'yoeito contains schisandra fruit, polygala root, and citrus unshiu peel, which all act on the respiratory system. A component of schisandra fruit, $\alpha$-cubebenoate, has been reported to suppress accumulation of eosinophils, macrophages, and lymphocytes in the pulmonary alveoli in a bronchial asthma model and inhibited Th2 cytokine and TGF- $\beta 1$ in pulmonary tissue (11). In a pneumonitis model, ninjin'yoeito was shown to suppress lung injury and death as well as production of nitric oxide in the serum (12). Pirfenidone is a therapeutic agent for IPF and has been shown to have an antifibrotic action in models of pulmonary fibrosis and hepatic cirrhosis $(13,14)$. Similarly, in a hepatic fibrogenesis model, ninjin'yoeito was found to suppress increases in the hepatic levels of hydroxyproline, which is an index of total collagen, as well as the production of cytokines that induce fibrogenesis (TGF- $\beta 1$ and IL-13) (15). Investigation of additional cases will help to clarify the effect of ninjin'yoeito for the treatment of respiratory diseases. We believe that our study makes a significant contribution to the literature because the findings showed that NYT can improve fatigue and contribute to maintaining quality of life in IP patients.

\section{DATA AVAILABILITY STATEMENT}

The original contributions presented in the study are included in the article/supplementary material, further inquiries can be directed to the corresponding author/s.

\section{ETHICS STATEMENT}

The studies involving human participants were reviewed and approved by Fukuoka University Hospital Ethics Committee. The patients/participants provided their written informed consent to participate in this study. Written informed consent was obtained from the individual(s) for the publication of any potentially identifiable images or data included in this article.

\section{AUTHOR CONTRIBUTIONS}

HK conducted the study and wrote the manuscript. HI and MF instructed the study. All authors contributed to the article and approved the submitted version.
4. Rolland Y, Perrin A, Gardette V, Filhol N, Vellas B. Screening older people at risk of malnutrition or malnourished using the simplified nutritional appetite questionnaire (SNAQ): a comparison with the mininutritional assessment (MNA) tool. J Am Med Dir Assoc. (2012) 13:314. doi: 10.1016/j.jamda.2011.05.003

5. Tanaka M, Fukuda S, Mizuno K, Imai-Matsumura K, Jodoi T, Kawatani $\mathrm{J}$, et al. Reliability and validity of the Japanese version of the Chalder Fatigue Scale among youth in Japan. Psychol Rep. (2008) 103:68290. doi: 10.2466/pr0.103.3.682-690

6. Chalder T, Berelowitz G, Pawlikowska T, Watts L, Wessely S, Wright D, et al. Development of a fatigue scale. J Psychosom Res. (1993) 37:14753. doi: 10.1016/0022-3999(93)90081-P 
7. Bestall JC, Paul EA, Garrod R, Garnham R, Jones PW, Wedzicha JA. Usefulness of the Medical Research Council (MRC) dyspnoea scale as a measure of disability in patients with chronic obstructive pulmonary disease. Thorax. (1999) 54:581-6. doi: 10.1136/thx.54.7.581

8. Shigekusa T, Tsubouchi H, Yanagi S, Nakazato M. Ninjin'yoeito improves cachexia in the respiratory system (in Japanese). Annal Japanese Respir Soc. (2019) 8:338.

9. Goswami C, Dezaki K, Wang L, Inui A, Seino Y, Yada T. Ninjin'yoeito activates ghrelin-responsive and unresponsive NPY neurons in the arcuate nucleus and counteracts cisplatin-induced anorexia. Neuropeptides. (2019) 75:58-61. doi: 10.1016/j.npep.2019.03.001

10. Yamada C, Mogami S, Kanno H, Nishi A, Shimobori C, Hattori T. Ninjin'yoeito ameliorates anorexia and decrease in the nest building via a dopamine D2 receptor-dependent manner in a novel murine apathy model (in Japanese). Japanese Pharmacol Ther. (2018) 46:207-15.

11. Shin JS, Ryu S, Cho YW, Kim HJ, Jang DS, Lee KT. Inhibitory effects of $\beta$-chamigrenal, isolated from the fruits of schisandrachinensis, on lipopolysaccharide-induced nitric oxide and prostaglandin E2 production in RAW 264.7 macrophages. Planta Med. (2014) 80:655-61. doi: 10.1055/s-0034-1368544

12. Tanaka K, Sawamura S. Therapeutic effect of a traditional Chinese medicine, ren-shen-yang-rong-tang (Japanese name: Ninjin'yoeito) on nitric oxidemediated lung injury in a mouse infected with murine cytomegalovirus. Int Immunopharmacol. (2006) 6:678-85. doi: 10.1016/j.intimp.2005.10.011
13. Schelegle ES, Mansoor JK, Giri S. Pirfenidone attenuates bleomycininduced changes in pulmonary functions in hamsters. Proc Soc Exp Biol Med. (1997) 216:392-7. doi: 10.3181/00379727-21644187

14. Tada S, Nakamuta $M$, Enjoji $M$, Sugimoto $R$, Iwamoto $H$, Kato $\mathrm{M}$, et al. Pirfenidone inhibits dimethylnitrosamine-induced hepatic fibrosis in rats. Clin Exp Pharmacol Physiol. (2001) 28:522-7. doi: 10.1046/j.1440-1681.2001.03481.x

15. Ochi T, Kawakita T, Nomoto K. Effects of Hochu-ekki-to and Ninjin'youeito, traditional Japanese medicines, on porcine seruminduced liver fibrosis in rats. Immunopharmacol Immunotoxicol. (2004) 26:285-98. doi: 10.1081/IPH-120037726

Conflict of Interest: The authors declare that the research was conducted in the absence of any commercial or financial relationships that could be construed as a potential conflict of interest.

Copyright (C) 2021 Kushima, Ishii and Fujita. This is an open-access article distributed under the terms of the Creative Commons Attribution License (CC BY). The use, distribution or reproduction in other forums is permitted, provided the original author(s) and the copyright owner(s) are credited and that the original publication in this journal is cited, in accordance with accepted academic practice. No use, distribution or reproduction is permitted which does not comply with these terms. 